\title{
BMJ Open Translation, adaptation and validation of two versions of the Chronic Liver Disease Questionnaire in Malaysian patients for speakers of both English and Malay languages: a cross- sectional study
}

Shasha Khairullah, Sanjiv Mahadeva

To cite: Khairullah S, Mahadeva S. Translation, adaptation and validation of two versions of the Chronic Liver Disease Questionnaire in Malaysian patients for speakers of both English and Malay languages: a crosssectional study. BMJ Open 2017;7:e013873. doi:10.1136/ bmjopen-2016-013873

- Prepublication history and additional material are available. To view these files please visit the journal online (http://dx.doi.org/ 10.1136/ bmjopen-2016-013873).

Received 21 September 2016 Revised 1 March 2017 Accepted 10 March 2017

\section{(a) CrossMark}

Division of Gastroenterology, Department of Medicine, Faculty of Medicine, University of Malaya, Kuala Lumpur, Malaysia

Correspondence to

Dr. Sanjiv Mahadeva;

sanjiv@ummc.edu.my

\section{ABSTRACT}

Objective We aimed to adapt, translate and validate the Chronic Liver Disease Questionnaire (CLDQ) in Malaysian patients with chronic liver diseases of various aetiologies. Setting Tertiary level teaching institution in Malaysia. Participants The validation process involved 211 adult patients (English language $n=101$, Malay language $\mathrm{n}=110$ ) with chronic liver disease. Characteristics of the study subjects were as follows: mean (SD) age was 56 (12.8) years, $58.3 \%$ were male and $41.7 \%$ female. The inclusion criteria were patients 18 years or older with chronic hepatitis and/or liver cirrhosis of any aetiology. The exclusion criteria were as follows: presence of hepatic encephalopathy, ongoing treatment with interferon and presence of other chronic conditions that have an impact on health-related quality of life (HRQOL).

Methods A cross-sectional study was conducted. Cultural adaptation of the English version of the CLDQ was performed, and a Malay version was developed following standard forward-backward translation by independent native speakers. Psychometric properties of both versions were determined by assessing their internal consistency, test-retest reliability and discriminant and convergent validity.

Results Cronbach's alpha for internal consistency across the various domains of the CLDQ was 0.95 for the English version and 0.92 for the Malay version. Test-retest analysis showed excellent reliability with an intraclass correlation coefficient of 0.89 for the English version and 0.93 for the Malay version. The average scores of both the English and Malay versions of the CLDQ demonstrated adequate discriminant validity by differentiating between non-cirrhosis (English 6.3, Malay 6.1), compensated cirrhosis (English 5.6, Malay 6.0) and decompensated cirrhosis (English 5.1, Malay 4.9) $(p<0.001)$. Convergent validity showed that correlation was fair between the English $(\rho=0.59)$ and Malay $(p=0.47)$ CLDQ versions with the $E Q-5 D$, a generic $H R Q 0 L$ instrument. Conclusion The English and Malay versions of the CLDQ are reliable and valid disease-specific instruments for assessing HRQOL in Malaysian patients with chronic liver disease.

\section{Strengths and limitations of this study}

- A translated and adapted version of the Chronic Liver Disease Questionnaire (CLDQ) has been found to be valid and reliable for assessing health-related quality of life in a multiracial Asian population with chronic liver disease.

- The validation cohort included patients with a varied aetiology of chronic liver disease.

- This study was performed at a single academic centre that may limit its applicability to other settings.

- The sample size was small, particularly that of patients with advanced chronic liver disease.

- This cross-sectional study was not able to assess responsiveness of the CLDQ.

\section{INTRODUCTION}

Chronic liver disease is one of the leading causes of mortality and morbidity worldwide. Recent reports have shown that deaths due to liver cirrhosis, chronic viral hepatitis and hepatic decompensation events worldwide have increased over the past 30 years, from 676000 ( $1.54 \%$ of global deaths) in 1980 to more than 1 million in 2010 (2\% of global deaths). ${ }^{1}$ In Malaysia, it is rapidly becoming one of the leading causes of disease-related mortality. Over the last 20 years, liver cirrhosis and liver cancer have increased in rank to become the 19th and 20th causes for premature mortality in Malaysia, respectively, based on a census report in 2010 . $^{2}$

Other manifestations of the disease are known to result in various debilitating symptoms. Fatigue, abdominal pain and nausea, depression, anxiety, inability to perform daily activities and vocational and interpersonal problems have all been reported. ${ }^{34}$ Even 
though some of these complications can be measured using standard clinical outcomes, most have no physical findings and are therefore poorly assessed by clinicians. There is increasing evidence that evaluating the patient's health-related quality of life (HRQOL) provides a more complete assessment of a patient's health status, by taking into consideration the complications that are difficult to measure using traditional clinical methods. ${ }^{5}$

Major advances have been made in the management of chronic liver disease. Some interventions like liver transplantation and antiviral therapy in hepatitis $\mathrm{C}$ infection are aimed for curative intent. However, treatment of decompensated cirrhosis, especially in the elderly, are mainly geared towards symptomatic relief, improving their quality of life and ability to function. Therefore, an objective assessment of the latter, such as the Chronic Liver Disease Questionnaire (CLDQ), is important in evaluating treatment outcome. ${ }^{5}$ This is particularly relevant in a country like Malaysia, where liver transplantation is not often practiced, resulting in a palliative approach towards patients with advanced liver disease. ${ }^{6}$ Younossi $e t$ al developed the first CLDQ after realising the need for a systematic assessment that is specifically designed for patients with chronic liver disease. ${ }^{7}$ To our knowledge, no existing HRQOL instrument has been validated for use in the Malaysian population.

\section{Objective}

We aimed to develop local English and Malay (the two most common languages spoken in Malaysia) versions of the CLDQ and validate both versions in Malaysian patients with chronic liver disease.

\section{METHODS}

\section{Subjects}

Ethical approval was obtained from our local institutional review board prior to the commencement of this study and complied with the latest version of the Declaration of Helsinki (University Malaya Medical Centre (UMMC) Ethics Reference no 1066.2). A cross-sectional study was conducted between January and August 2015. Consecutive adult patients with chronic liver disease who attended the gastroenterology clinic, including those who required admission under the gastroenterology team at UMMC were invited to participate in the study. Informed and written consent were obtained. A total of 211 patients were interviewed, of which 123 patients were male and the remaining 88 patients were female.

The inclusion criteria for subjects in this study were as follows: patients 18 years or older with chronic hepatitis, or liver cirrhosis of any aetiology or primary hepatocellular carcinoma. Chronic hepatitis was defined by an elevation of serum transaminases 1.5 times above the upper limit of normal for longer than 6 months for patients with non-viral hepatitis. The diagnosis for chronic hepatitis B was based on serology (HBsAg and anti-HBcIgG). Chronic hepatitis $\mathrm{C}$ was diagnosed on the presence of hepatitis $\mathrm{C}$ antibodies and detectable viral load on PCR. Causes for chronic liver disease were divided into viral hepatitis (hepatitis B or hepatitis C), alcohol, non-alcoholic fatty liver disease (NAFLD) and others. The patients were grouped into those without cirrhosis (viral hepatitis carriers, fibrosis and fatty liver changes), compensated cirrhosis (Child-Pugh A cirrhosis) and decompensated cirrhosis (Child-Pugh B and C cirrhosis and hepatocellular carcinoma).

The exclusion criteria for subjects in this study were as follows : age $<18$ years, psychiatric disorders (on prescribed medication for psychiatric disorders), presence of hepatic encephalopathy, ongoing treatment with interferon (due to physical and psychological side effects), presence of other chronic conditions that have an impact on HRQOL that include heart failure, malignancies (except for hepatocellular carcinoma), chronic renal failure, chronic obstructive pulmonary disease and inflammatory bowel disease, and inability to communicate in English or Malay.

\section{HRQOL instruments}

The EQ-5D is an established generic HRQOL instrument comprising five items on mobility, self-care, pain, usual activities and psychological status with three answers for each question ( $1=$ no problem, $2=$ moderate problems, $3=$ severe problems). ${ }^{8}$ An overall utility score is calculated based on these domains ranging from 0 (worse health scenario) to a maximum of 1 (best case scenario). A visual analogue scale is used to assess general health status with 100 indicating the best health state. The English and Malay versions of the EQ-5D have been validated for use in the Malaysian population. ${ }^{9}$

The CLDQ is a specific HRQOL questionnaire consisting of 29 questions assessed on a 7-point Likert scale whereby responses range from 'All of the time' to 'none of the time'. ${ }^{7}$ Each of the responses are graded from 1 to 7 . The domains assessed include abdominal symptoms, fatigue, systemic symptoms, activity, emotional function and worry. The questions are randomly distributed. The CLDQ results are obtained from a scale of 1-7 by dividing each domain score with the number of questions it contains. ${ }^{7}$ Permission to use the questionnaire was obtained from the original developer prior to the commencement of the study.

Patients were interviewed in English or Malay, depending on their language of proficiency. Both the CLDQ and the EQ-5D were administered in one setting. Additional clinical and demographic information were obtained from the interview in the form of a questionnaire as well as from medical records.

\section{Translation and adaptation of the English and Malay versions of the CLDQ}

Cross-cultural adaptation of the English version of CLDQ was performed in 10 healthy subjects with various educational backgrounds. In-depth cognitive interviews were conducted to determine its suitability in Malaysian adults. 
Alterations were made to the original English version if particular words or phrases were not understood.

The original version was translated into Malay using standard forward-backward translation. Two independent forward translations (from English to Malay) by two native Malay speakers were performed. Subsequently, a reconciliation of both versions were done with differences resolved through discussion. A backward translation (from Malay to English) was then produced.

\section{Validation of the English and Malay versions of the CLDQ}

Psychometric properties of the Malaysian English and Malay versions of the CLDQ were evaluated by assessing reliability, discriminant validity and convergent validity. ${ }^{1011}$ The reliability of an instrument is assessed by internal consistency and test-retest. Internal consistency evaluates the correlation across items in different domains and across all items of a questionnaire. Test-retest reliability, an assessment of error caused by repeated measurement under a similar condition, was performed by a repeat telephone interview 2-4 weeks after the baseline interview. Discriminant validity refers to the ability of an instrument to discriminate between the varying severities of a particular disease. In this case, the discriminant validity of the CLDQ was assessed by its ability to distinguish between scores for non-cirrhosis, compensated and decompensated cirrhosis. Convergent validity refers to how well a particular instrument correlates with domains of an existing HRQOL questionnaire. In this study, convergent validity of the CLDQ was assessed by correlating its total score against the utility score of the EQ-5D, an established generic HRQOL.

\section{Statistical analysis}

Data were expressed as means with $\mathrm{SD}$, or proportions where appropriate. Internal consistency was evaluated using Cronbach's alpha analysis, with an acceptable level of $>0.70$. Test-retest reliability was assessed using intraclass correlation coefficients (ICC), with a desired value of $>0.70$. Discriminant validity of scores among different stages of chronic liver disease was obtained using one-way analysis of variance. Convergent validity of the CLDQ domains was analysed against the EQ-5D using Spearman's correlation. ${ }^{10}$ Strong, fair and weak correlations were defined as $>0.60,0.30-0.60$ and $<0.30$, respectively. ${ }^{12}$ Statistical significance for hypothesis fulfilment was defined as a $p$ value of $<0.05$. All analyses were carried out using SPSS for Windows (V.22.0).

\section{RESULTS}

\section{Adaptation of the Malaysian English CLDQ and development of the Malay CLDQ}

Cognitive debriefing of the original English version of the CLDQ was conducted on 10 healthy English-speaking subjects from various educational background. Minor changes were made to the original questionnaire. The opening statement of each question 'How much of the time' was altered to 'How many times'. Part of item 14 was rephrased from 'bothered by a limitation of your diet' to 'bothered by your loss of appetite'.

A Malay version of the CLDQ was produced according to the protocol outlined above. Cognitive debriefing of the translated version was performed on 10 healthy subjects of various educational background, all of Malay ethnicity. No difficulties in understanding the phrasing of the questions were reported by all 10 subjects. No further alterations were made prior to use in the validation study.

During the cognitive debriefing for both the English and Malay versions, some subjects did comment on the Likert scale components being too similar and that this might cause some confusion. We came up with another alternative using numbers ranging from 0 (none of the time) to 6 (all the time). It took most patients a shorter amount of time to understand the questionnaire with the additional explanation of a number scale. On average, patients completed both the CLDQ and the EQ-5D in 10 min, making the CLDQ a suitable instrument to be used on an outpatient basis (see online supplementary appendix).

\section{Patient characteristics}

Two hundred and eleven patients with chronic liver disease were interviewed between January 2015 and August 2015. One hundred and one patients were interviewed in English, and 110 patients were interviewed in Malay. There were no missing data. Their clinical and social demographic data are summarised in table 1 . The mean age of patients were younger in all groups for those who were Malay speaking in comparison with those who were English speaking. Ethnicity varied in both language categories-patients interviewed in Malay were predominantly ethnic Malays $(65.5 \%)$, while patients interviewed in English were predominantly ethnic Chinese (72\%). In both groups, patients with cirrhosis were older (mean age of patients without cirrhosis was 56.8 years vs mean age of patients with cirrhosis was 64.1 years), either unemployed or retired and had lower education levels than those without cirrhosis. Viral hepatitis was the main cause for chronic liver disease, with a total of $48 \%$ with hepatitis B and $14 \%$ with hepatitis C. This observation is consistent with previous studies that had reported on the aetiology of chronic liver disease in Malaysia. ${ }^{13} 14$ However, NAFLD was more common among ethnic Malays (11\% ethnic Malays vs $5.6 \%$ ethnic Chinese). Chronic hepatitis C was found to be more common among ethnic Malays as well, again, consistent with findings from previous reports. ${ }^{13}$

\section{Reliability}

In the Malaysian English CLDQ Cronbach's alpha demonstrated values for each CLDQ domain as follows: abdominal symptoms: 0.89 , fatigue: 0.88 , systemic symptoms: 0.88 , activity: 0.90 , emotional function: 0.88 and worry: 0.90. In the Malay CLDQ, Cronbach's alpha values for similar domains were as follows: abdominal symptoms $: 0.85$, fatigue: 0.81 , systemic symptoms: 0.83 , activity: 0.85 , emotional function: 0.82 and worry: 0.85 . 
Table 1 Characteristics and demography of Malaysian patients with chronic liver disease in the study

English speaking $\mathrm{n}=101$

Malay speaking $\mathrm{n}=110$

\begin{tabular}{|c|c|c|c|c|c|c|}
\hline & $\begin{array}{l}\text { Non-cirrhosis } \\
\mathrm{n}=62\end{array}$ & $\begin{array}{l}\text { Compensated } \\
\text { cirrhosis } n=31\end{array}$ & $\begin{array}{l}\text { Decompensated } \\
\text { cirrhosis } n=8\end{array}$ & $\begin{array}{l}\text { Non-cirrhosis } \\
n=64\end{array}$ & $\begin{array}{l}\text { Compensated } \\
\text { cirrhosis } n=29\end{array}$ & $\begin{array}{l}\text { Decompensated } \\
\text { cirrhosis } n=17\end{array}$ \\
\hline Mean age (SD) & $56.8(12.7)$ & $63.5(9.6)$ & $64.8(11.7)$ & 48.6 (12.9) & $58.2(8.7)$ & $59.2(10.6)$ \\
\hline Male:female & $31: 31$ & $17: 14$ & $5: 3$ & $39: 25$ & $23: 6$ & $8: 9$ \\
\hline \multicolumn{7}{|l|}{ Ethnicity (n (\%)) } \\
\hline Malay & $8(12.9 \%)$ & $2(6.5 \%)$ & $1(12.5 \%)$ & $41(64.1 \%)$ & $21(72.4 \%)$ & $10(58.8 \%)$ \\
\hline Chinese & $48(77.4 \%)$ & $19(61.3 \%)$ & $6(75.0 \%)$ & $18(28.1 \%)$ & $5(17.2 \%)$ & $1(5.9 \%)$ \\
\hline Indian & $5(8.1 \%)$ & 7 (22.6\%) & $1(12.5 \%)$ & $5(7.8 \%)$ & $3(10.3 \%)$ & $6(35.3 \%)$ \\
\hline Others & $1(1.6 \%)$ & $3(9.7 \%)$ & $0(0.0 \%)$ & $0(0.0 \%)$ & $0(0.0 \%)$ & $0(0.0 \%)$ \\
\hline \multicolumn{7}{|c|}{ Education level (n (\%)) } \\
\hline Primary & $1(1.6 \%)$ & $1(3.2 \%)$ & $1(12.5 \%)$ & $10(15.6 \%)$ & $8(27.6 \%)$ & $7(41.2 \%)$ \\
\hline Secondary & $25(40.3 \%)$ & $19(61.3 \%)$ & $6(75.0 \%)$ & $37(57.8 \%)$ & $17(58.6 \%)$ & $10(58.8 \%)$ \\
\hline Tertiary & $36(58.1 \%)$ & $11(35.5 \%)$ & $1(12.5 \%)$ & $17(26.6 \%)$ & $4(13.8 \%)$ & $0(0.0 \%)$ \\
\hline \multicolumn{7}{|c|}{ Employment status (n (\%)) } \\
\hline Employed & $31(50.0 \%)$ & 7 (22.6\%) & $2(25.0 \%)$ & $44(68.8 \%)$ & $10(34.5 \%)$ & $3(17.6 \%)$ \\
\hline $\begin{array}{l}\text { Unemployed/ } \\
\text { homemaker }\end{array}$ & $5(8.1 \%)$ & $3(9.7 \%)$ & $2(25.0 \%)$ & $6(9.5 \%)$ & $3(10.3 \%)$ & $5(29.4 \%)$ \\
\hline Retired & $26(41.9 \%)$ & $21(67.7 \%)$ & $4(50.0 \%)$ & $14(21.9 \%)$ & $16(55.2 \%)$ & $9(52.9 \%)$ \\
\hline \multicolumn{7}{|c|}{ Causes of chronic liver disease ( $(\%))$} \\
\hline Hepatitis B & $48(77.6 \%)$ & $9(29.0 \%)$ & $5(62.5 \%)$ & $27(42.2 \%)$ & $9(31.0 \%)$ & $4(23.5 \%)$ \\
\hline Hepatitis C & $4(6.5 \%)$ & $6(19.4 \%)$ & $2(25.0 \%)$ & $9(14.1 \%)$ & $5(17.2 \%)$ & $5(29.4 \%)$ \\
\hline Alcoholism & $1(1.6 \%)$ & $6(19.4 \%)$ & $0(0.0 \%)$ & $1(1.6 \%)$ & $3(10.3 \%)$ & $3(17.6 \%)$ \\
\hline NAFLD/NASH & $6(9.7 \%)$ & $4(12.9 \%)$ & $0(0.0 \%)$ & $27(42.2 \%)$ & $7(24.1 \%)$ & $0(0.0 \%)$ \\
\hline Others & $3(4.8 \%)$ & $6(19.5 \%)$ & $1(12.5 \%)$ & $0(0.0 \%)$ & $5(17.2 \%)$ & $5(29.4 \%)$ \\
\hline \multicolumn{7}{|c|}{ Child-Pugh classification } \\
\hline Child-Pugh A & $31(30.7 \%)$ & & & $29(26.3 \%)$ & & \\
\hline Child-Pugh B & $6(5.9 \%)$ & & & $10(9.1 \%)$ & & \\
\hline Child-Pugh C & $2(2.0 \%)$ & & & $7(6.4 \%)$ & & \\
\hline
\end{tabular}

NAFLD, non-alcoholic fatty liver disease; NASH, non-alcoholic steatohepatitis.

Ninety-nine $(98 \%)$ patients from the English group and $80(73 \%)$ patients from the Malay group participated in the follow-up telephone interview, which was conducted between 2 and 4 weeks after the first interview. In the Malaysian English CLDQ ICC between baseline and follow-up summary scores was 0.89 (95\% CI 0.84 to $0.93)$, demonstrating excellent test-retest reliability. In the Malay CLDQ ICG between baseline and follow-up summary scores was 0.93 (95\% CI 0.88 to 0.95 ), similarly demonstrating excellent test-retest reliability. The demographics of Malay-speaking subjects who responded to the second interview were compared with the original sample. We found no differences in mean age, gender, ethnicity, education level, employment status nor in severity of chronic liver disease between the two groups (data as online supplementary table).

\section{Discriminant validity}

There was a significant decrease in domain scores from patients without cirrhosis to patients with decompensated cirrhosis in both the English and Malay versions of the CLDQ. The CLDQ in English and Malay was found to have good discriminant validity (table 2 and table 3 ).

\section{Convergent validity}

For the English version of the CLDQ, correlation of the EQ-5D utility score with the overall CLDQ score was $0.59(\mathrm{p}<0.001)$. For the Malay version of the CLDQ, correlation of the EQ-5D utility score with the overall CLDQ score was $0.47(\mathrm{p}<0.001)$.

\section{DISCUSSION}

Psychometric properties of both Malaysian English and Malay versions of the CLDQ were well tested. Reliability testing of both versions demonstrated satisfactory consistency of its various domains and excellent testretest reliability. Discriminant validity analysis revealed that both versions of the CLDQ were able to differentiate between non-cirrhosis, compensated cirrhosis 
Table 2 Discriminant validity among different groups of patients in the English language version of CLDQ (mean \pm SD)

\begin{tabular}{llllc}
\hline CLDQ domains & No cirrhosis $(\mathbf{n}=62)$ & $\begin{array}{l}\text { Compensated cirrhosis } \\
(\mathbf{n}=31)\end{array}$ & $\begin{array}{l}\text { Decompensated cirrhosis } \\
(\mathbf{n}=8)\end{array}$ & p Value \\
\hline Abdominal symptoms & $6.6(0.8)$ & $5.8(1.6)$ & $4.8(1.8)$ & $<0.001$ \\
\hline Fatigue & $6.0(1.0)$ & $5.5(1.5)$ & $4.8(1.8)$ & 0.038 \\
Systemic symptoms & $6.4(0.8)$ & $5.8(1.3)$ & $5.4(1.2)$ & 0.003 \\
Activity & $6.2(1.1)$ & $5.4(1.6)$ & $5.5(1.8)$ & 0.014 \\
\hline Emotional function & $6.4(0.9)$ & $5.6(1.4)$ & $5.2(1.4)$ & 0.001 \\
Worry & $6.5(0.9)$ & $5.5(1.5)$ & $4.9(1.7)$ & $<0.001$ \\
\hline Average CLDQ & $6.3(0.7)$ & $5.6(1.2)$ & $5.1(1.4)$ & $<0.001$ \\
\hline
\end{tabular}

CLDQ, Chronic Liver Disease Questionnaire.

and decompensated cirrhosis satisfactorily. Convergent validity analysis demonstrated that the English and Malay CLDQ domains (some more than others) correlated well with an established generic HRQOL, the EQ-5D.

\section{Comparison between the Malaysian English and the Malay CLDQ}

There were some differences noted between the adapted English and the Malay versions of the CLDQ. With regards to discriminant validity, the English version showed significant discriminant validity throughout all six domains (table 2). In the Malay version, the values for the domain 'worry' was found not to be significant (table 3). In the Malay version, the correlation value for convergent validity was lower than the English version. It is likely that the lower education levels among the Malay-speaking patients may have resulted in poorer comprehension of some of the questions pertaining to healthcare domains in the HRQOL instruments.

\section{Comparison with other validation studies}

The CLDQ has been validated and translated into other languages worldwide. Figure 1 is a bar chart comparing the domain and overall scores of the CLDQ between our study and seven other studies. ${ }^{15-21}$ The patients in our study appear to have higher CLDQ scores in comparison with the Thai, Korean, Indian, Chinese, Spanish, Serbian and Japanese studies (figure 1). This may be due to several factors. First, only a small proportion of our study subjects had decompensated cirrhosis. Second, the lower educational levels of our study subjects ,particularly in the Malay-speaking group, may have led to an element of ignorance among many patients about their disease or state of condition. Lastly, differences in socio-cultural attitudes to health, known to differ among populations, may be responsible for the observed differences in CLDQ scores between our study and the rest. ${ }^{22} 23$

Convergent validity of the CLDQ into other languages were mostly done with the SF-36, another generic HRQOL instrument. ${ }^{7161824}$ The SF-36 has 36 questions and is known to take longer to complete. ${ }^{25}$ We decided to use the EQ-5D in this Malaysian study for its simplicity and brevity, similar to the Korean and Chinese validation studies. ${ }^{17} 19$ Although brief, the EQ-5D has been shown to be a valid and reliable instrument for evaluating HRQOL in various diseases. ${ }^{26} 27$

One of the notable differences in this study related to question 29 of the CLDQ, which enquired about the availability of a liver organ if a transplant was needed. Almost all patients (except for one) were not aware that such an option existed for patients with end-stage liver failure. This was a clear reflection of the aforementioned status of liver transplantation in this country. ${ }^{6}$ Although an overwhelming majority selected 'none of the time' as their answer, the item was not omitted in lieu of the potential development of liver transplant services in the future.

Table 3 Discriminant validity among different groups of patients in the Malay language version of CLDQ (mean \pm SD)

\begin{tabular}{llllr}
\hline CLDQ domains & No cirrhosis $(\mathbf{n}=\mathbf{6 4})$ & $\begin{array}{l}\text { Compensated cirrhosis } \\
(\mathbf{n}=\mathbf{2 9})\end{array}$ & $\begin{array}{l}\text { Decompensated cirrhosis } \\
(\mathbf{n}=\mathbf{1 7})\end{array}$ & p Value \\
\hline Abdominal symptoms & $6.1(1.3)$ & $6.1(1.4)$ & $4.1(1.6)$ & $<0.001$ \\
\hline Fatigue & $5.6(1.1)$ & $5.5(1.6)$ & $4.4(1.5)$ & 0.006 \\
Systemic symptoms & $6.3(0.8)$ & $6.0(1.1)$ & $5.3(1.4)$ & 0.002 \\
Activity & $6.5(0.9)$ & $6.0(1.4)$ & $4.5(1.7)$ & $<0.001$ \\
\hline Emotional function & $6.1(0.8)$ & $5.8(1.2)$ & $5.4(1.3)$ & 0.015 \\
Worry & $6.2(1.0)$ & $6.2(1.0)$ & $5.8(1.0)$ & 0.314 \\
\hline Average CLDQ & $6.1(0.7)$ & $6.0(1.0)$ & $4.9(1.0)$ & $<0.001$ \\
\hline
\end{tabular}

CLDQ, Chronic Liver Disease Questionnaire. 


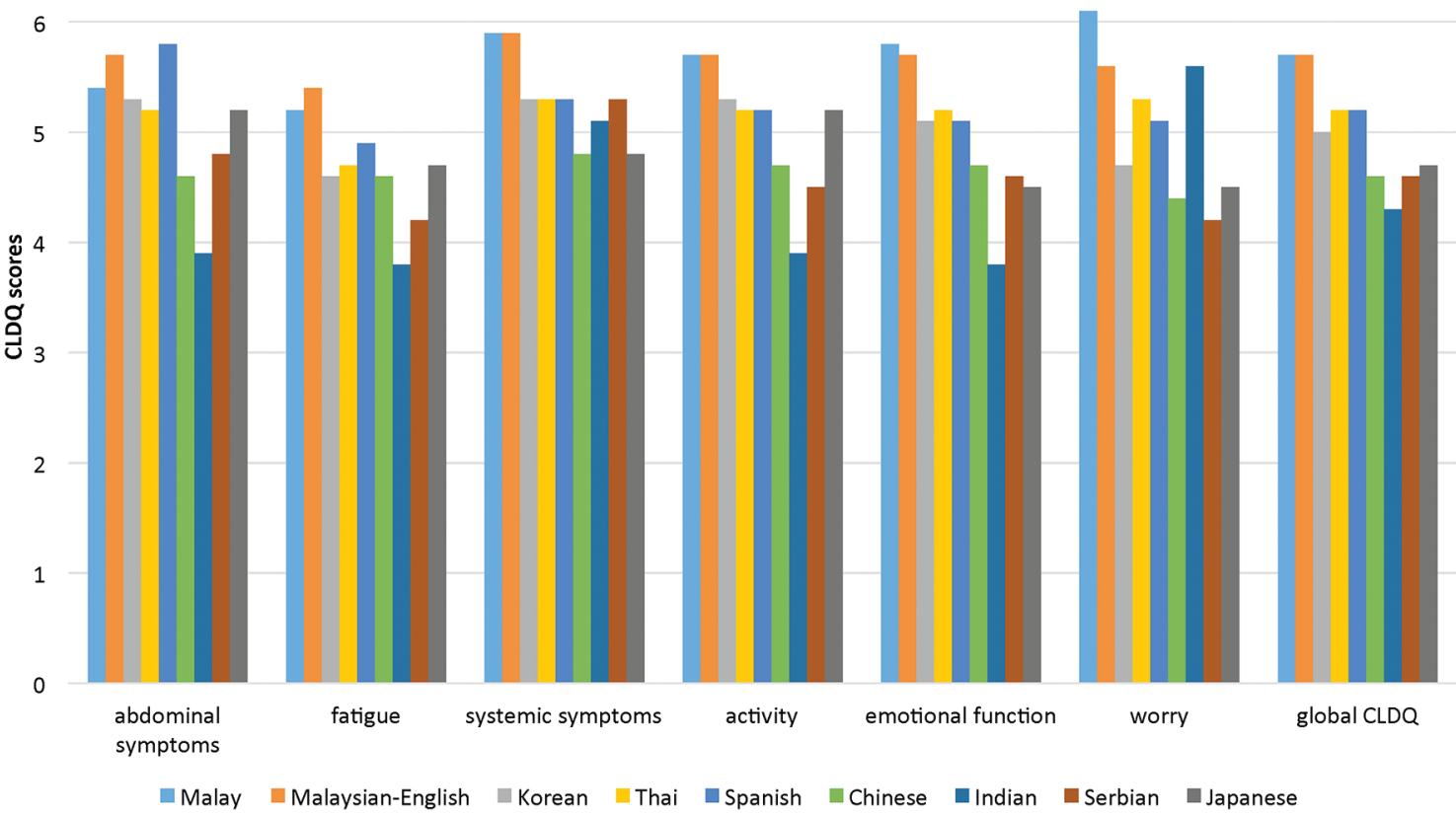

Figure 1 CLDQ scores in other countries. CLDQ, Chronic Liver Disease Questionnaire.

\section{Strengths and limitations}

The high level of completion of data among the patients indicates good acceptability of both the English and Malay CLDQ versions. The questionnaire was easily understood with most patients completing it within $10 \mathrm{~min}$. Data from this validation study were comparable with CLDQ validation studies in different populations. This study also included a wide range of patients with chronic liver disease severity, from non-cirrhosis to those with decompensated (Child-Pugh C) cirrhosis and hepatocellular carcinoma.

One of the limitations to the study was its relatively small sample size, particularly of patients with decompensated cirrhosis. We believe this was one of the main reasons the overall CLDQ domain scores were higher than in other studies. Furthermore, this study was based in a single tertiary care centre, which may have resulted in a selection bias of study subjects. However, as previously mentioned, the demography and clinical characteristics of our subjects were consistent with previous publications from this country. ${ }^{13} 14$ Although we have tried to exclude patients with diagnosed psychiatric disorders, we may have inadvertently included participants with untreated psychological disorders in this study. However, we feel that this may not have represented a major confounder to this QOL validation study, in view of the good correlation with liver disease severity observed.

Another limitation related to the differences in interview techniques administered for test-retest reliability. The second interview was telephone based compared with the initial face-to face method, which may have resulted in a lower ICC value. Nevertheless, it was unlikely that any major change in the patient's HRQOL could have happened within the interval of 2-4 weeks as the patients were stable and were not experiencing acute decompensation at the time of interview.

Finally, this study was not able to assess the responsiveness of the CLDQ in our patients. It is important to note that these issues should be addressed in future studies.

\section{CONCLUSION}

Overall, the CLDQ in both the English and Malay languages appear to be acceptable and easily understood among Malaysian patients with chronic liver disease. Both versions have been shown to be reliable and valid for assessing HRQOL in adults with chronic liver disease. Our data are consistent with other studies exploring the validity and reliability of the CLDQ in chronic liver disease. It is anticipated that the locally developed CLDQ will be useful in the assessment and clinical management of patients with chronic liver disease in this country.

Contributors SM planned the study. SK and SM researched data, conducted the statistics and wrote the manuscript.

Competing interests None declared.

Ethics approval Medical Ethics Committee University Malaya Medical Centre.

Provenance and peer review Not commissioned; externally peer reviewed.

Data sharing statement Additional data that is not available online can be obtained by contacting the corresponding author.

Open Access This is an Open Access article distributed in accordance with the Creative Commons Attribution Non Commercial (CC BY-NC 4.0) license, which permits others to distribute, remix, adapt, build upon this work non-commercially, and license their derivative works on different terms, provided the original work is properly cited and the use is non-commercial. See: $\mathrm{http} / / /$ creativecommons.org/licenses/by-nc/4.0/ 
(C) Article author(s) (or their employer(s) unless otherwise stated in the text of the article) 2017. All rights reserved. No commercial use is permitted unless otherwise expressly granted.

\section{REFERENCES}

1. Mokdad AA, Lopez AD, Shahraz S, et al. Liver cirrhosis mortality in 187 countries between 1980 and 2010: a systematic analysis. BMC Med 2014;12:145.

2. GBD Profile: Malaysia. Global burden of diseases, injuries, and risk factors study 2010. $2010 \mathrm{http}: / /$ www.healthdata.org/sites/default/ files/files/country_profiles/GBD/ihme_gbd_country_report_malaysia. pdf

3. Bianchi G, Marchesini G, Nicolino F, et al. Psychological status and depression in patients with liver cirrhosis. Dig Liver Dis 2005;37:593-600.

4. McDonald J, Jayasuriya J, Bindley P, et al. Fatigue and psychological disorders in chronic hepatitis C. J Gastroenterol Hepatol 2002;17:171-6.

5. Younossi ZM, Guyatt G. Quality-of-life assessments and chronic liver disease. Am J Gastroenterol 1998;93:1037-41.

6. Hooi LS, Mansor LY. Seventh report of the National Transplant Registry. Kuala Lumpur: National Transplant Registry, 2010:3-12.

7. Younossi ZM, Guyatt G, Kiwi M, et al. Development of a disease specific questionnaire to measure health related quality of life in patients with chronic liver disease. Gut 1999;45:295-300.

8. Brooks R. EuroQol: the current state of play. Health Policy 1996;37:53-72.

9. Mahadeva S, Wee HL, Goh KL, et al. The EQ-5D (Euroqol) is a valid generic instrument for measuring quality of life in patients with dyspepsia. BMC Gastroenterol 2009;9:20.

10. Fayers PM, Machin D. Wiley Online Library . Quality of life: assessment, analysis and interpretation. Hoboken, New Jersey: John Wiley \& Sons, Ltd, 2010.

11. Terpos E, Christoulas D. Effects of proteasome inhibitors on bone Cancer. Bonekey Rep 2013;2:395.

12. Chan YH. Biostatistics 104: correlational analysis. Singapore Med J 2003;44:614-9.

13. Qua CS, Goh KL. Liver cirrhosis in Malaysia: peculiar epidemiology in a multiracial Asian country. J Gastroenterol Hepatol 2011;26:1333-7.

14. Merican I, Guan R, Amarapuka D, et al. Chronic hepatitis B virus infection in Asian countries. J Gastroenterol Hepatol 2000;15:1356-61.
15. Ray I, Dutta D, Basu P, et al. Quality of life assessment of patients with chronic liver disease in eastern India using a Bengali translation chronic liver disease questionnaire. Indian J Gastroenterol 2010;29:187-95.

16. Sobhonslidsuk A, Silpakit C, Kongsakon R, et al. Chronic liver disease questionnaire: translation and validation in Thais. World $J$ Gastroenterol 2004;10:1954-7.

17. Choi HM KS, Kim Y, et al. Validation of the korean version of the chronic liver disease questionnaire (CLDQ) for the assessment of health-related quality of life in korean patients with chronic hepatitis $B, 2008$.

18. Ferrer M, Córdoba J, Garin O, et al. Validity of the spanish version of the chronic liver disease questionnaire (CLDQ) as a standard outcome for quality of life assessment. Liver Transp/ 2006;12:95-104.

19. Che YH, You J, Chongsuvivatwong V, et al. Dynamics and liver disease specific aspects of quality of life among patients with chronic liver disease in Yunnan, China. Asian Pac J Cancer Prev 2014;15:4765-71.

20. Popovic DDj, Kovacevic NV, Kisic Tepavcevic DB, et al. Validation of the chronic liver disease questionnaire in Serbian patients. World $J$ Gastroenterol 2013;19:4950-7.

21. Tanaka A, Kikuchi K, Miura R, et al. Validation of the Japanese version of the Chronic Liver Disease Questionnaire for the assessment of health-related quality of life in patients with chronic viral hepatitis. Hepatol Res 2016;46:E45-50.

22. Ariff KM, Beng KS. Cultural health beliefs in a rural family practice: a Malaysian perspective. Aust J Rural Health 2006;14:2-8.

23. Yap P, Mahadeva S, Goh KL. The influence of cultural habits on the changing pattern of functional dyspepsia. Dig Dis 2014;32:217-21.

24. Rucci P, Taliani G, Cirrincione L, et al. Validity and reliability of the Italian version of the Chronic Liver Disease questionnaire (CLDQ-I) for the assessment of health-related quality of life. Dig Liver Dis 2005;37:850-60.

25. McHorney CA, Ware JE, Raczek AE. The MOS 36-Item Short-Form health survey (SF-36): II. psychometric and clinical tests of validity in measuring physical and mental health constructs.1993;31:247-63.

26. Schrag A, Selai C, Jahanshahi M, et al. The EQ-5D--a generic quality of life measure-is a useful instrument to measure quality of life in patients with Parkinson's disease. J Neurol Neurosurg Psychiatry 2000;69:67-73.

27. Luo N, Chew LH, Fong KY, et al. Validity and reliability of the EQ-5D self-report questionnaire in Chinese-speaking patients with rheumatic diseases in Singapore. Ann Acad Med Singapore 2003;32:685-90. 